universe was indeed a live issue of the period, and a highly contentious one - appearing, for example, in the Papal edict of 1277 that banned a list of scientific teachings. But it was a debate that Grosseteste apparently chose to avoid. None of his surviving treatises discusses the possibility of other forms of universe, however close he came to implying it in his cosmogony.

Of course we know now, thanks to telescope observations from the early seventeenth century onwards, that a geocentric cosmos is untenable. But in 1225, it was the simplest theory consistent with the observations. Grosseteste's effort to give a physical account of its origin is an impressive achievement, but it also reminds us of the limitations of our own current cosmological theory, with its reliance on intangible factors such as 'dark matter' and 'dark energy'.

\section{SCIENTIFIC VALUE}

The translation of De Luce is an exemplar of the importance of collaborations between the arts and sciences, of thinking and learning together in new ways, and a reminder that the intellectual tradition we now call science has a long and rich history.

Both the scientists and the humanities scholars in our collaboration found working together enriching and transformational; it forced us to engage with different ideas and problems. There were challenges: getting used to each other's methodologies and approaches took time and patience. And our expectations changed. At the start of the project we had hoped for a sharper understanding of the text; we were surprised when new science emerged as well.

What next for the collaboration? The Durham-led team has examined three of Grosseteste's science works in detail so far. There are at least another ten to explore, including a work on the origin of sounds (De Generatione Sonorum). The scientific writings of Grosseteste's immediate predecessors, Alfred of Sareshull and Alexander Neckham, and his successors, including Bacon, could hold similar insights into the evolution of ideas.

Funding for such interdisciplinary work, however, remains a problem. In the United Kingdom, none of the scientific research councils offered grants for such a project. In the end, we were funded by the Arts and Humanities Research Council. The US granting system is similarly biased. The European Research Council and philanthropic sources, such as the Wellcome Trust, do fund sciencearts projects, but in our experience it is easier to obtain funding for science and social science collaborations than for science and humanities partnerships.

Because projects such as ours can be of significant scientific and cultural value, scientific granting agencies should consider funding arts and sciences projects or partnering with arts and humanities councils to translate other early scientific works, for example.

The eight-century journey from Grosseteste's cosmological ideas to our own offers a rich illustration of the slow evolution in our understanding, and of the delight to be found in reaching out into nature with our imagination.

Tom C. B. McLeish is in the Department of Physics, Durham University, UK. His book Faith and Wisdom in Science is published in May. Richard G. Bower and Brian K. Tanner are in the Department of Physics, Durham University, UK. Hannah E. Smithson is in the Department of Experimental Psychology, University of Oxford, UK. Cecilia Panti is in the Department of Corporate Governance Philosophy, University of Rome Tor Vergata, Italy. Neil Lewis is in the Department of Philosophy, Georgetown University, Washington DC, USA. Giles E. M. Gasper is in the Institute of Medieval and Early Modern Studies and in the Department of History, Durham University, UK.

e-mail:t.c.b.mcleish@durham.ac.uk

1. Bower, R. G. et al. Preprint at http://arxiv.org/ abs/1403.0769 (2014).

2. Smithson, H. E. etal. J. Opt. Soc. Am. 29, A346-A352 (2012).

3. Smithson, H. E. et al. J. Opt. Soc. Am. 31, A341-A349 (2014).

\title{
The art of science advice to government
}

\author{
Peter Gluckman, New Zealand's chief science adviser, offers his ten \\ principles for building trust, influence, engagement and independence.
}

I n 2009, I was appointed as the first science adviser to the Prime Minister of New Zealand. The week I was appointed coincided with the government announcement that the New Zealand food industry would not be required to add folate to flourbased products to help to prevent neuraltube defects in newborns, despite an earlier agreement to do so. As it happens, this is an area of my own scientific expertise and, before my appointment, I had advised the government that folate supplementation should occur. But various groups had stirred considerable public concern on the matter, about health risks and about medicalizing the food supply.
Thus, in my first media interview as science adviser I was asked how I felt about my advice not being heeded. I pointed out that despite strong scientific evidence to support folate supplementation, a democratic government could not easily ignore overwhelming public concern about the food supply. The failure here was not political; rather, it was the lack of sustained and effective public engagement by the medicalscience community on the role of folate in the diet. As a result, the intervention did not get the social licence necessary to proceed.

Five years on, I am still in the post. I have come to understand that the primary functions and greatest challenges for a science adviser are providing advice not on straightforward scientific matters, but instead on issues that have the hallmarks of what has been called post-normal science ${ }^{1}$. These issues are urgent and of high public and political concern; the people involved hold strong positions based on their values, and the science is complex, incomplete and uncertain. Diverse meanings and understandings of risks and trade-offs dominate.

Examples include the eradication of exogenous pests in New Zealand's unique ecosystems, offshore oil prospecting, legalization of recreational psychotropic drugs, water quality, family violence, obesity, teenage morbidity and suicide, the ageing 
$>$ population, the prioritization of earlychildhood education, reduction of agricultural greenhouse gases, and balancing economic growth and environmental sustainability.

My own experience is of a Westminsterstyle parliamentary democracy in a small advanced economy. In this context, I have distilled a set of ten principles to guide my work. Other countries have different forms of government and different cultural histories of public reason; highlevel scientific advice may be provided by individuals, councils or academies, or a combination. Nevertheless, I think my

"Policy-
makers
and elected
officials
rightly
guard their
responsibility
to define
policy."
guidelines are relevant to all those providing advice to senior levels of government.

These principles differ a little from those that might guide individual researchers and academics in attempting to influence policy in areas of their own interest and expertise $^{2,3}$. Crucially, science advisers are obliged to advise in the context of the policy process. This means elucidating the evidence-informed options, rather than simply advocating a course of action.

\section{TOP TEN}

Maintain the trust of many. The science adviser must sustain in parallel the trust of the public, the media, policy-makers, politicians and the science community. This is especially true in times of crisis and is no small challenge. Food-safety panics such as foot-and-mouth disease and Creutzfeldt-Jakob disease (CJD) catalysed a strengthening of the science-advisory system in the United Kingdom, enhancing the roles of departmental science advisers. The aftermath of the 2011 nuclear meltdown in Fukushima is causing Japanese officials to take a critical look at advisory practices ${ }^{4}$.

In my case, it was an earthquake that tested trust. In early 2011, Christchurch in New Zealand experienced the second of two major earthquakes in six months. The events had devastating consequences, including nearly 200 deaths, and effectively destroyed our second-largest city. This cluster of quakes was unusual and led to seismologists publicly competing in their interpretation of the nature of the fault-lines and future risks. This confused the public and policy-makers.

It took considerable dialogue with the scientists for them to understand the need for simple and consistent communication, and to accept that erudite and, in many ways, self-serving scholarly discourse did not belong on the front page of newspapers every day. What was needed was clear communication of the knowns and unknowns.
Worse, because the earthquake happened on the day of a full Moon, a popular astrologer got primetime coverage when he predicted another big earthquake would occur one month later when the Moon and Sun would next be in alignment. Panic set in. We worked with New Zealand's Science Media Centre to calm the public while acknowledging seismic uncertainty.

Protect the independence of advice. The advisory role should be structured so as to protect its independence from both political interference and premature filtering in the policy process. There is inevitably a tension between such independent advice and departmental policy processes, and it takes considerable diplomacy to create a trusted partnership between an external adviser and departmental officials. The terms of my appointment protect my independence in that I continue to be an employee of my academic institution, seconded to the prime minister, and my advisership is not tied to the electoral cycle. That said, an adviser must recognize that publicly disturbing the democratic process could mean losing the trust of the elected leader and thus any potential for influence. So there are issues on which a national scientific academy or a panel may be best placed to advise or to be seen supporting the individual adviser.

Report to the top. Scientific advice must be available directly - uncensored - to the head of government or the head of the relevant department. Indeed, the questions for which advice is most often sought tend to be politically sensitive and cut across individual portfolios.
In New Zealand, for instance, the economic importance of land-based primary industries must be balanced against maintaining our ecosystems and the eco-tourism industry built on them. These concerns are the responsibility of separate ministries, whose respective takes on environmental impact are inevitably framed by their mandates. The adviser's perspective transcends these.

Distinguish science for policy from policy for science. Science advising is distinct from the role of administering the system of public funding for science. There is potential for perceived conflict of interest and consequent loss of influence if the science adviser has both roles. There is a risk that the adviser comes to be perceived as a lobbyist for resources, or that the role becomes restricted to the ministry that manages the national research funding. Yes, a science adviser should have input into science policy, but there is a delicate balance to strike.

Early in my appointment, an unnecessary tension was created by media portrayals that I, rather than the relevant ministry, was the key influence on science policy. This is not so — nor did I want it to be - but communication with that ministry became strained for some time, denting my effectiveness.

Expect to inform policy, not make it. Science advice is about presenting a rigorous analysis of what we do and do not know. Alone, it does not make policy. There are many other appropriate inputs to policy, including fiscal considerations and public opinion. Policy-makers and elected officials rightly guard their responsibility to define policy - and this means choosing between

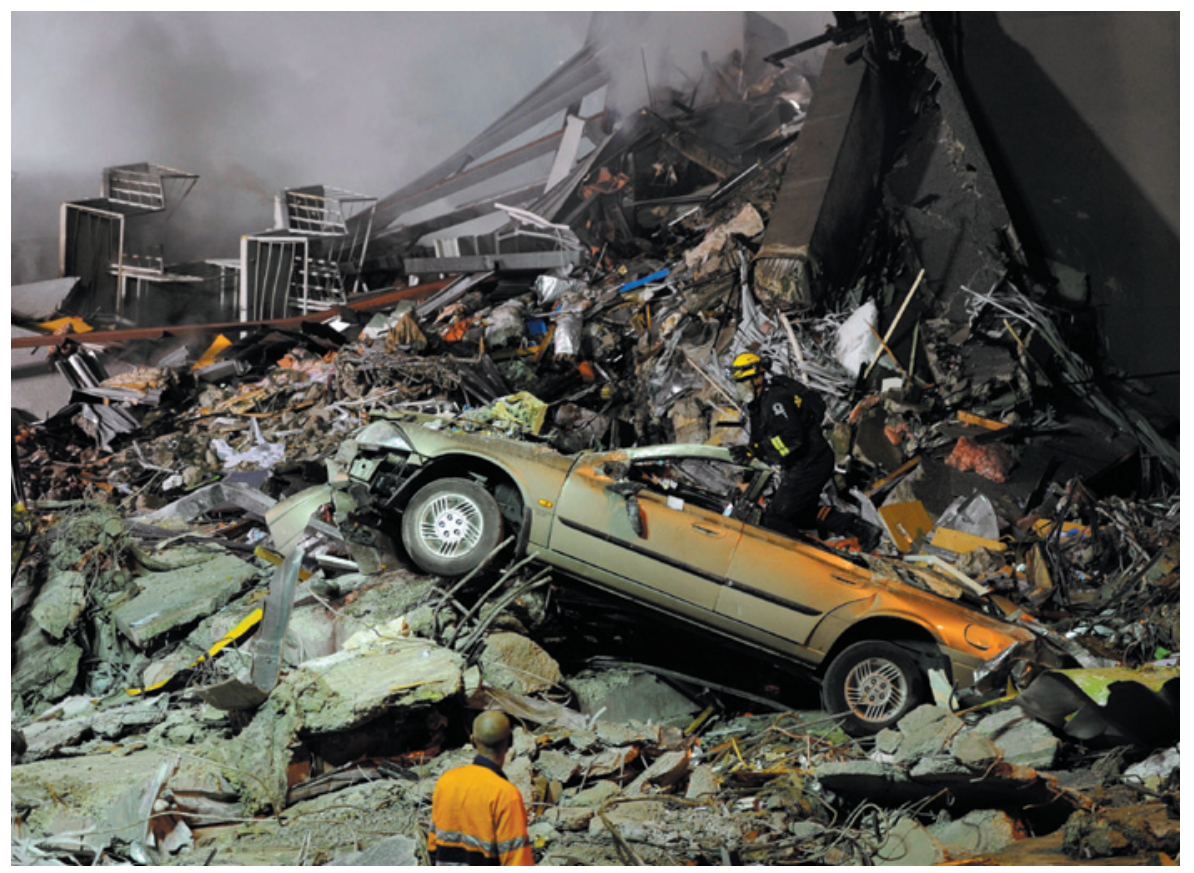

Post-normal science: Christchurch, New Zealand, after its second earthquake in six months. 


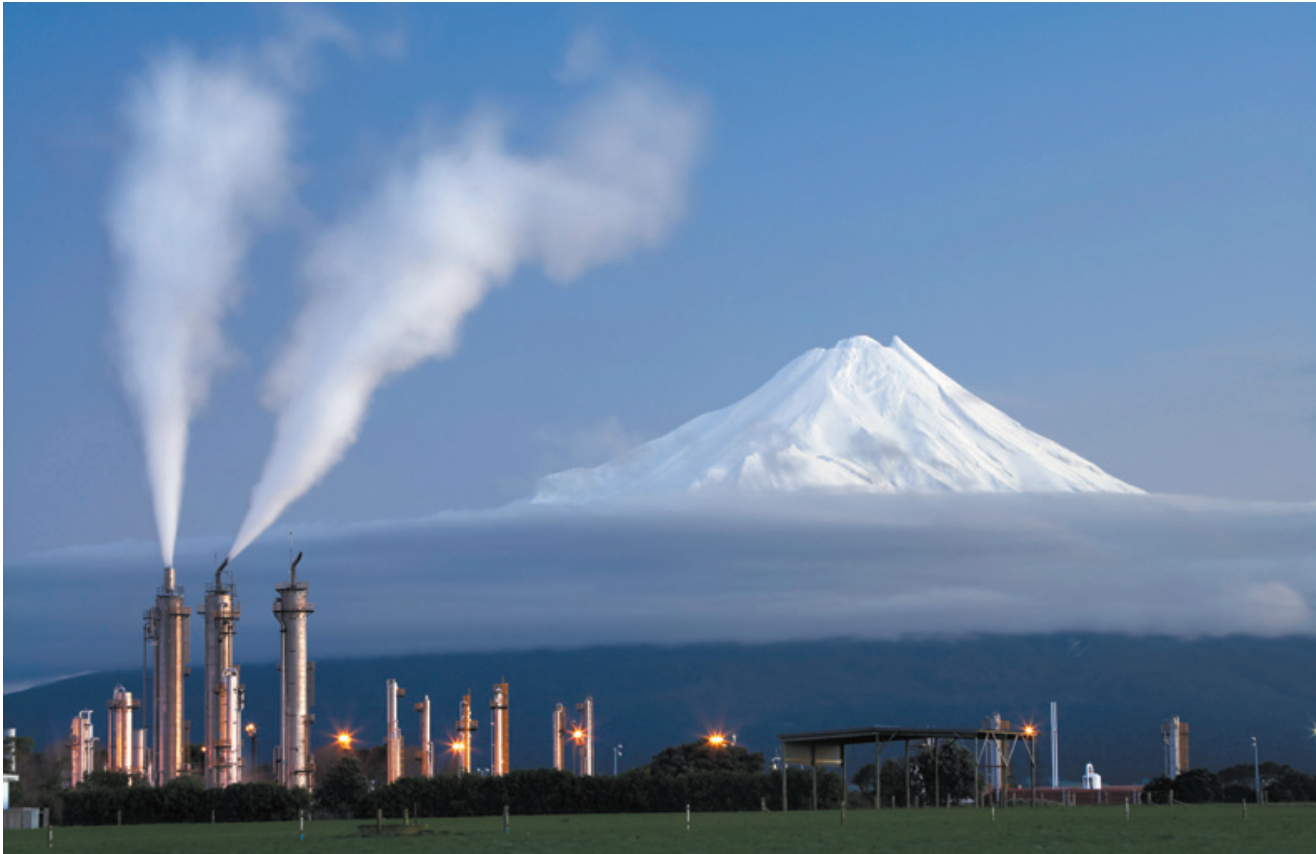

A gas-production station near Mount Taranaki in New Zealand's North Island.

options with different trade-offs. This is not the domain of a science adviser. Being explicit about this ${ }^{5}$ has eased my capacity to establish and sustain trust broadly across government and the policy community.

Give science privilege as an input into policy. While acknowledging the other relevant inputs into policy formation, we need to demonstrate why science should hold a privileged place among the 'types of knowledge' that may be meaningful to a politician. These include social tradition and popular belief. The privilege of science-derived knowledge comes from its set of standard procedures - for example, replication and peer review - that limit the influence of beliefs and dogma. The other inputs into policy are valueintensive, and rightly so.

Recognize the limits of science. Science can increasingly address complex questions over which policy-makers and elected officials agonize. But scientists must not overstate what is or can be known, even though the shift from a view of science as a source of certainty to a source of probability can frustrate and confuse decision-makers and the public. How many politicians or issues advocates have claimed that they can find a scientist to back any position as, indeed, at least one did in the folate debate? This attitude reflects the dangerous temptation to use science to justify value-based beliefs ${ }^{6}$ and a lack of literacy about what science is (a process $)^{7}$. For example, much of the debate about climate change is not primarily about the data. Rather, it is about intergenerational economic interests.
Act as a broker not an advocate. Trust can be earned and maintained only if the science adviser or advisory committee acts as a knowledge broker, rather than as an advocate $^{6}$ - often a subtle distinction. When formal science advice is perceived as advocacy, trust in that advice and in the adviser is undermined, even if the advice is accepted. For example, exaggerated presentations about the causes of storms and floods can erode the credibility of the underlying argument about global warming. My own academic research has been focused on the developmental origins of obesity, and I have had to be careful ensure a balance of advice. Even so, where there is strong advocacy for other approaches, suspicion about the balance of my advice is hard to avoid (see go.nature.com/syxyee).

Engage the scientific community. The science adviser must know how to reach out to scientists for the appropriate expertise, and help them to enact their social responsibility in making their knowledge accessible and understandable, and in being more self-aware about when they might be acting as advocates. These issues are encapsulated in the recently updated, groundbreaking Code of Conduct for Scientists ${ }^{8}$, which directly implies a distinction between brokerage and advocacy, published by the Japanese Council of Science.

Engage the policy community. The role of the science adviser is often less about providing direct technical expertise than it is about nudging attitudes and practices to enhance both the demand for and the supply of evidence for public policy.

Why? Because sceptics in the policy community are surprisingly prevalent. In 2012, I surveyed how our public-service personnel use evidence in making policy ${ }^{4}$. Several ministries stated that their job was to design policy that met the minister's requirements, not to advise on policy options on the basis of available evidence. Studies in Canada and Australia ${ }^{9}$ found similar results.

\section{GOOD ADVICE}

These principles that guide my own work probably apply to most models of science advice. The use of advisers, advisory councils or academies need not be mutually exclusive. Different approaches suit different purposes and are the product of a country's culture, history, political and social structures and approaches to civic reason ${ }^{10}$.

In my experience, achieving the culture change that encourages the better use of scientifically derived evidence in government relies on a level of trust that may be best achieved by one-to-one relationships with senior members of the executive government. In crises, such relationships are essential. By contrast, for complex and chronic issues, I believe that advisory committees or academies have a crucial part to play.

Happily, these matters are increasingly being discussed. In August this year, the first global conference of academics and practitioners of science advice to governments will take place in Auckland, New Zealand (see www.globalscienceadvice.org). I hope that two days of discussion between thought leaders from around the world about principles, methods, tensions and solutions from myriad contexts will make an important contribution to this rapidly growing field.

Peter Gluckman is chief science adviser to the Prime Minister of New Zealand. He is also professor at the Liggins Institute, University of Auckland, New Zealand. e-mail:csa@pmcsa.org.nz

1. Funtowicz, S. O. \& Ravetz, J. R. Futures 25, 739-755 (1993).

2. Sutherland, W. J., Speigelhalter, D. \& Burgman, M. A. Nature 503, 335 (2013)

3. Douglas, H. Science, Policy and the Value-Free Ideal (Univ. Pittsburgh Press, 2009).

4. Doubleday, R. \& Wilsdon, J. Nature 485, 301-302 (2012).

5. Gluckman, P. D. Towards Better Use of Evidence in Policy Formation: A Discussion Paper (Office of the Prime Minister's Science Advisory Committee, 2011); available at http://go.nature.com/ntrw7u.

6. Pielke, R. A. Jr The Honest Broker: Making Sense of Science in Policy and Politics (Cambridge Univ. Press, 2007).

7. Gluckman, P. D. The Role of Evidence in Policy Formation and Implementation (Office of the Prime Minister's Science Advisory Committee, 2013); available at http://go.nature.com/ kmxmbx.

8. Science Council of Japan Statement: Code of Conduct for Scientists - Revised Version (SCJ, 2013); available at http://go.nature.com/nhrnbb.

9. Hickey, G. M., Forest, P., Sandall, J. L., Lalor, B. M. \& Keenan, R. J. Sci. Pub. Pol. 40, 529-543 (2013).

10.Jasanoff, S. Science and Public Reason (Routledge, 2012). 